\title{
Research Paper \\ Reliability and Factor Structure of Social Paradigm Belief Inventory
}

\author{
Mohammad Mehrad Sadr ${ }^{1}$, Seyed Sepehr Hashemian"1, Hanieh Khoshsima², "Saharnaz Akhbari
}

1. PhD Student in Psychology, Department of Psychology, Faculty of Psychology and Education, Allameh Tabataba'i University, Tehran, Iran.

2. MA in Clinical Psychology, Department of Clinical and Health Psychology, Faculty of Psychology and Education, Shahid Beheshti University, Tehran, Iran.

3. MA in Clinical Psychology, Department of Psychology, Faculty of Psychology and Education, Allameh Tabataba'i University, Tehran, Iran.

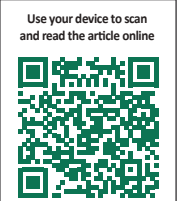

Citation: Mehrad Sadr M, Hashemian SS, Khoshsima H, Akhbari S. [Reliability and Factor Structure of Social Paradigm Belief Inventory (Persian)]. Iranian Journal of Psychiatry and Clinical Psychology. 2018; 24(3):340-355. http:// dx.doi.org/10.32598/ijpcp.24.3.340

http://dx.doi.org/10.32598/ijpcp.24.3.340

Funding: See Page 352 (c) Copyright: The Author(s)

Received: 14 Apr 2018 Accepted: 27 May 2018

Keywords: Adult, Cognition, Factor analysis, Surveys and questionnaire, Students

\section{A B STRACT}

Objectives This study aimed to investigate the factor structure and reliability of the Social Paradigm Belief Inventory (SPBI).

Methods A total of 381 students of Hakim Sabzevari University were selected by stratified cluster random sampling method and responded to SPBI in 2016. This research is descriptive with a validation method. Exploratory and confirmatory factor analysis were used to investigate factor structure of the instrument, and Cronbach $\alpha$ and test-retest method were employed for examining the reliability.

Results Results of principal component with varimax rotation showed that 4-actorial and 6-factorial solutions explained $30 \%$ and $38 \%$ of whole variance, respectively. The results of the confirmatory factor analysis showed that the 6-factorial solution was a better indication of the data than 4-factorial solutions and it better fits the data in Iran society. The Cronbach $\alpha$ value of the instrument was 0.7 and as for the subscales of the instrument, it varied from 0.54 to 0.71 and the correlation coefficient of test-retest was 0.72 that showed acceptable reliability for the instrument.

Conclusion Enjoying an acceptable reliability and fitting well with the data in Iran society make the 6 -factor SPBI a suitable measure for determining adults' cognitive development for screening or clinical purposes.

\section{Extended Abstract}

\section{Introduction}

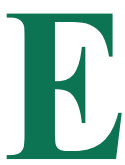

ven the most daring pioneering researchers find it difficult to find out if recognition of their growth in adulthood continues through qualitative organizational distinct levels. Another Piaget needed to accurately observe the characteristics of the argument model in adults when solving problems of everyday life. The seeds of such an attempt have already been planted in a remarkable study; in this study Perry traced the growth of thinking among Harvard students. Perry (1968) quotes that the growth of an adult and dialectical way of thinking implies the recognition of the relativism of all knowledge, which leads to a revolutionary change in intellectual and moral thinking.

Researchers have studied adolescents and adults such as Ericsson, Fisher, Harvey, Hunt and Screwdog, Kitchener

* Corresponding Author:

Saharnaz Akhbari, MA

Address: Department of Psychology, Faculty of Psychology and Education, Allameh Tabataba'i University, Tehran, Iran.

Tel: +98 (21) 44070068

E-mail: akhbari.saharnaz@gmail.com 
and King, Kelberg, Levinger and Perry; Researchers are particularly interested in writing models such as Rigel and Basschell that see dialectical thinking as an extension of the operation of ultrasound. Each of these models observes that humans grow in their thinking during their adult life, meaning that intellectual growth is not complete in adolescence. In fact, intellectual growth is a process of life, although its manifestations are very different from the context and the social environment to the context and the social environment, and it depends very much on individual differences. Although there are differences in these models, they all have a common trajectory, which is to follow the path from simplicity and absolutism to complexity and relativism, from objectivity to abstraction, and from regulating external behavior to internal behavioral regulation.

Since the mode of thinking in absolute, relativistic or dialectical way, as previously mentioned, is related to various variables such as identity, role conflict and emotional regulation, etc., it plays an important role in the various aspects of the life of individuals in There are individual, communicative and emotional spheres. Considering that thinking about adult growth theory after formal thinking in adolescence is determined, the cause of people's failures in understanding different issues and their subsequent behavior beyond adolescence can be at the level of their cognitive development In the adult search.

Because maladaptation usually start from adolescence and youth, and do not change over time, causing a person's displeasure or interruptions in some of his functions. This involves addressing the stages and stages of cognitive development. The study of the level of cognitive development in people is a tool to quantify and measure it. Such tools have already been developed to measure cognitive development, but none have Persian version. One of these tools, as discussed earlier, is the Cramer and colleagues' social ideals questionnaire. The purpose of this study is to investigate the factor structure of the Persian version of the social science beliefs questionnaire and to examine the internal consistency of the scale in the Iranian society.

\section{Method}

The present study investigated the factor structure and reliability of SPBI. A total of 381 students of Hakim Sabzevari University filled out the instrument. Exploratory and confirmatory factor analyses were used to investigate the factor structure of the instrument, and Cronbach's alpha and test-retest method were employed for examining the reliability (Table 1).

\section{Results}

Although the internal consistency and the test-retest method for the Likert spectrum have not been measured by Kramer et al., The results of this study are related to the overall reliability of the scale and the subscales of the Likert spectrum questionnaire with the results of Kramer and Associates are consistent with the overall consistency of the scale and sub-scales of the questionnaire. However, contrary to these, there is little difference in the present study between the internal coherence of the elements of relativism and dialectical thinking with other factors. The results of the test-retest method are consistent with the reliability of the questionnaire with the results of Kramer et al. on the existence of a high degree of test-retest reliability on the scale.

Table 1. Demographic characteristics of the study sample

\begin{tabular}{|c|c|c|c|}
\hline & & Frequency & Percentage \\
\hline \multirow{3}{*}{ Gender } & Man & 189 & 49.6 \\
\hline & & & \\
\hline & Woman & 192 & 50.4 \\
\hline \multirow{3}{*}{ Marriage } & Single & 341 & 89.5 \\
\hline & & & \\
\hline & Married & 40 & 10.5 \\
\hline \multirow{3}{*}{ Education } & B.A. & 284 & 74.5 \\
\hline & M.A. & 93 & 24.4 \\
\hline & Ph.D. & 4 & 1 \\
\hline
\end{tabular}




\section{Discussion}

Finally, it can be stated that this questionnaire has a relative reliability and factor analysis is the main factors of the questionnaire, and in general it can be used to recognize cognitive development in adults and to use screening and clinical. Limitations: It should be remembered that due to the limited sample of this study, as well as the use of the self-report tool and with regard to cultural differences, the generalization of results should be made with caution.

It is suggested that this study be conducted on other samples with a greater variety and size, in order to test the repeatability of the results. It is also suggested that future studies be interviewed to complete and compare the results of the questionnaire and also to compare the validity of the structure, the current questionnaire is compared with similar and different questionnaires.

\section{Ethical Considerations}

\section{Compliance with ethical guideline}

All the participants signed the informed consent of the research.

Funding

This research did not receive any specific grant from funding agencies in the public, commercial, or not-forprofit sectors.

\section{Conflict of interest}

There is no conflict of interest in this research.

\section{Acknowledgements}

We really appreciate all those who participated in the study. 


\title{
هايايى و ساختار عاملى يرسش نامه عقايد اصول اجتماعى
}

محمد مهراد صدر 'ه، سيد سيهر هاشميان'، حانيه خوشسيما'، "سحرناز اخبارى"

\author{
ا - انشجوى دكتراى روانشئاسى، كروه روانشئاسى، دانشكده روانشأسى و علوم تربيتى، دانشكاه علامه طباطبايي، تهران، ايران.

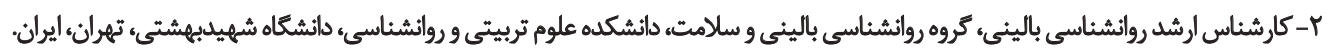

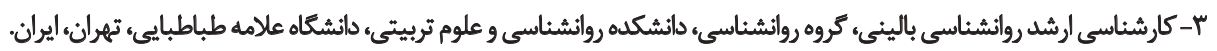

\begin{abstract}
حكبد
اهدافت مطالعه حاضر يك تحقيق توصيفى و از نوع اعتبارسئجى است. هدف اين مطالعه بررسى ساختار عاملى و بايائي يُرسشئامه عقايد اصول

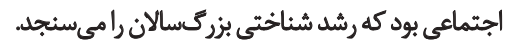

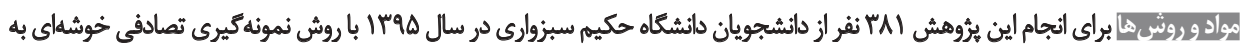

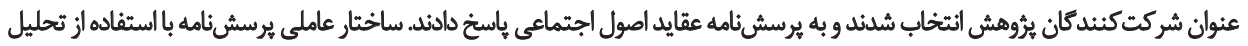

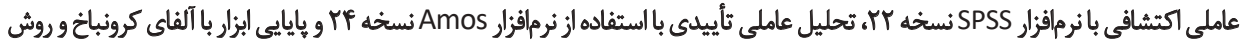

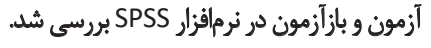

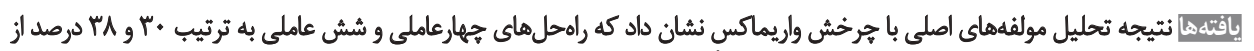

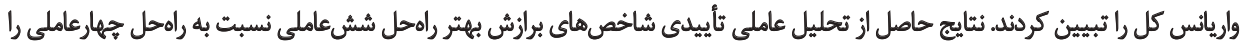

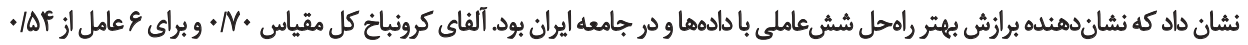

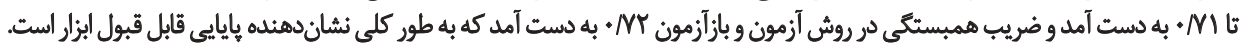

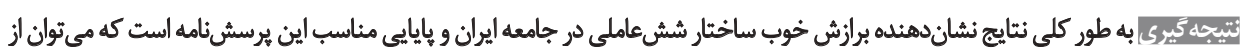

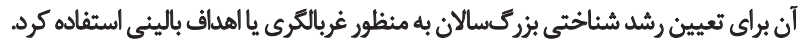

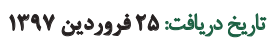

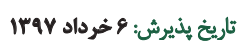

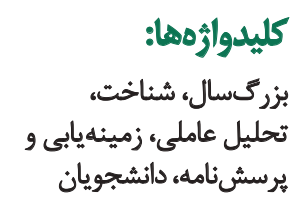

كليدوازوها:

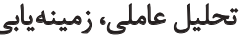
يرسشنامه، دانشجويان تينان

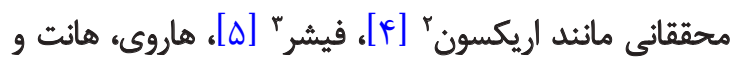

dale

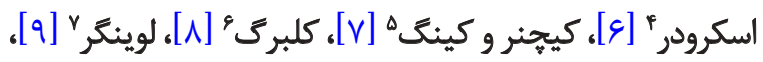

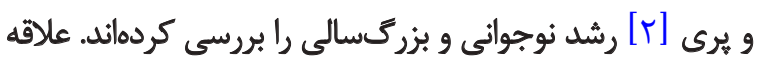

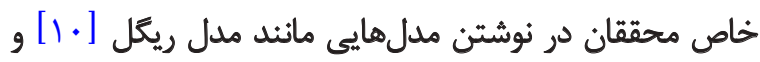

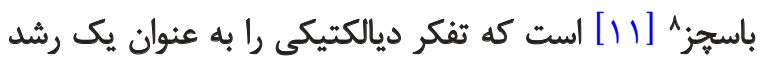

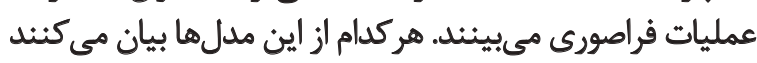

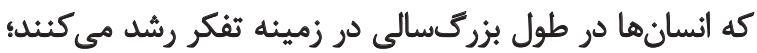

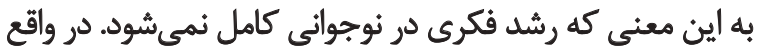

\footnotetext{
2. Erikson

3. Fischer

4. Harvey, Hunt, Schroder

5. Kitchener \& King

6. Kohlberg

7. Loevinger

8. Basseches
}

حتى جسورترين محققان بيرىشناسى نيز در يافتن جواب

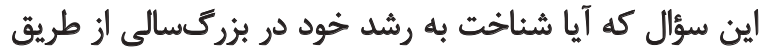

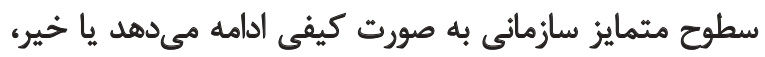

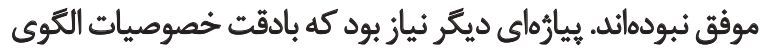

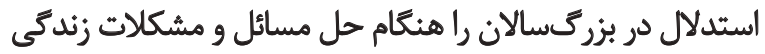

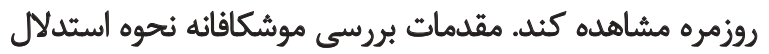

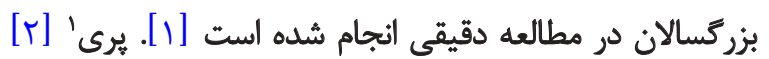

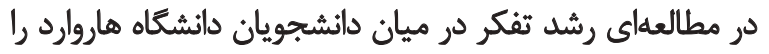

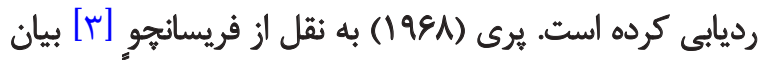

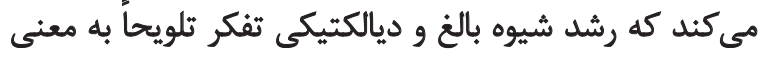

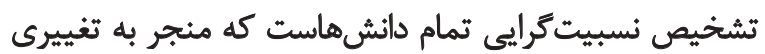
انقلابي در تفكر عقلاتي و اخلاقي مي تمام دانشود.

1. Perry

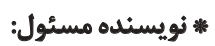

سعرناز اخهبارى

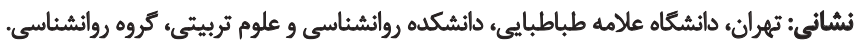

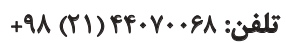

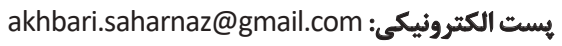




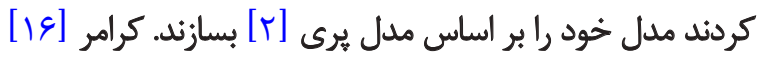

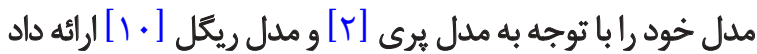

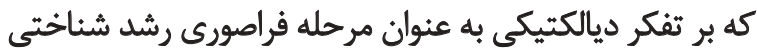

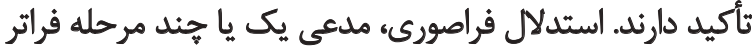

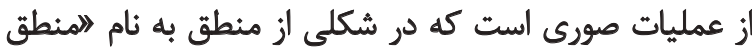

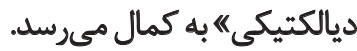

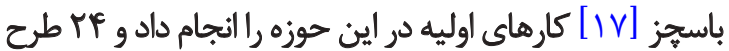

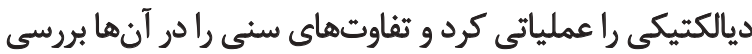

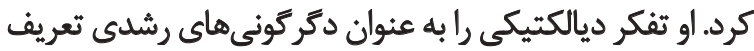

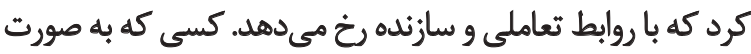

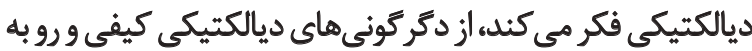

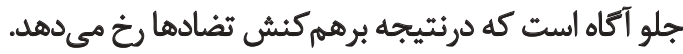
كرامر و همكاران [1 ] كار در زمينه تفكر ديالكتيكى راكسترش

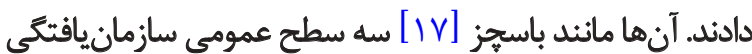

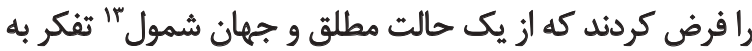

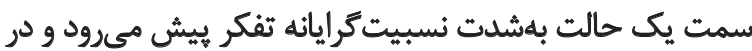

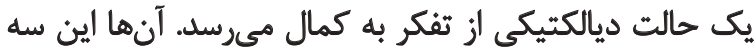

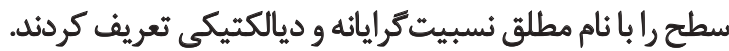

كسى كه در سطح تفكر مطلق قرار دارد، به منظور فهرم جهان

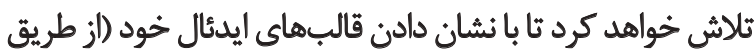

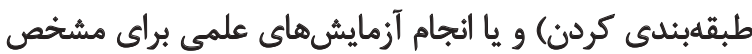

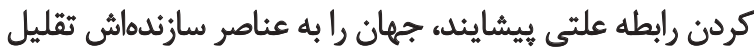

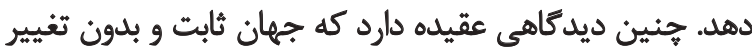

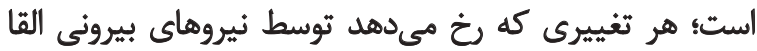

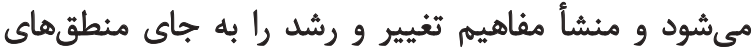

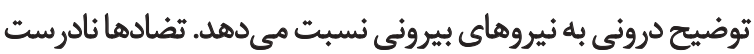

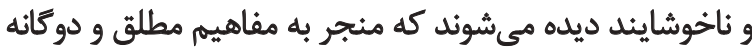

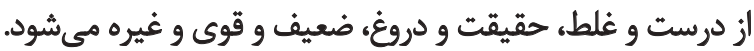

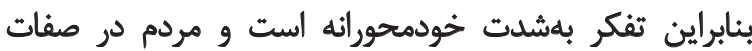

مشخصى كنجانده مي شوند كه قابل تغيير نيست [ [1 ]. در تفكر نسبيت كرا، تمام دانش متأثر از زمينهاش ديده مي شئود

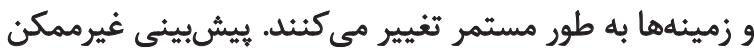

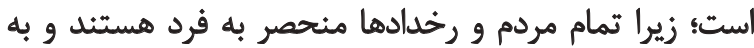

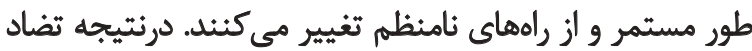

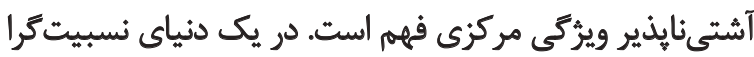

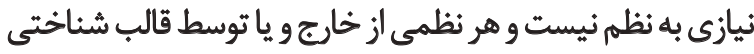

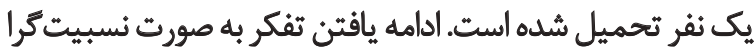

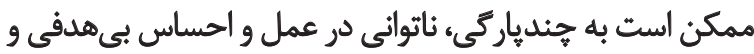

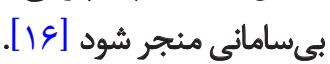

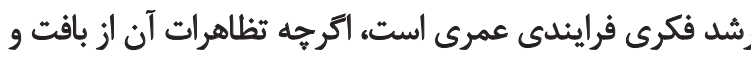

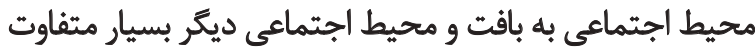

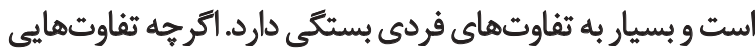

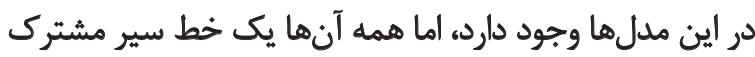

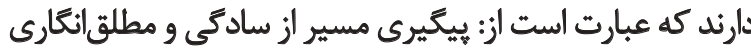

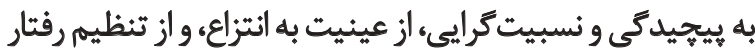

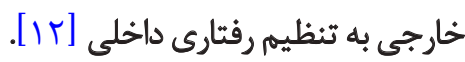
تئورىهاى رشد دانشجويان در تلاش هستند تا راهى را كه

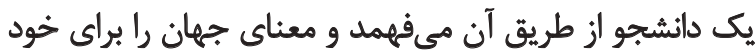

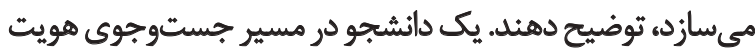

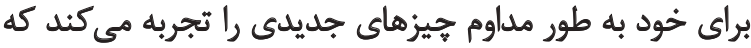

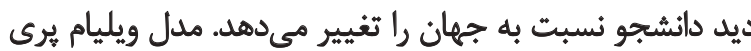

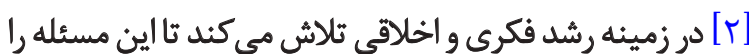

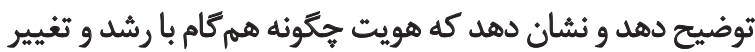

$$
\text { توانايىهاى فكرى شكل مى نيرد. }
$$

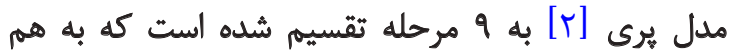

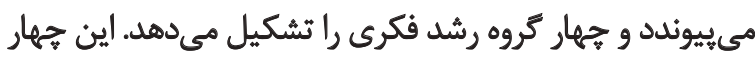

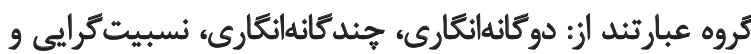

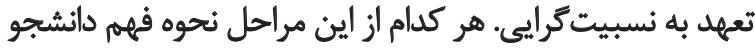

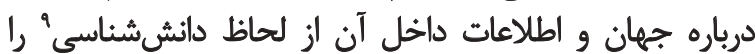

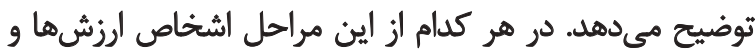

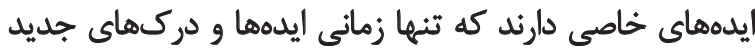

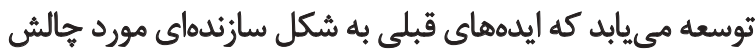

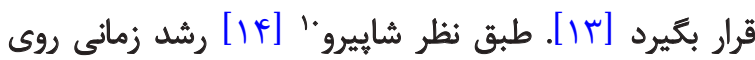

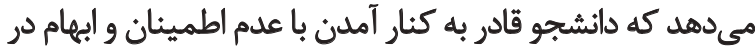

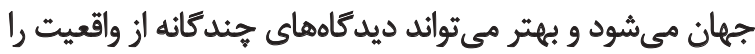

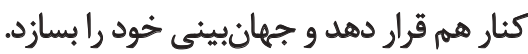

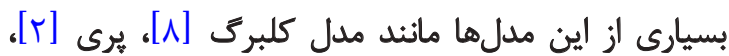
كيجنر و كينَ [V] به دليل انجام آزمونهاى بسيار موشكافيانه

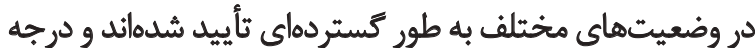

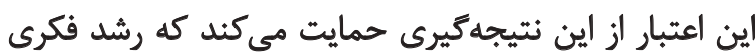
بزركسالان بديدهاى است كه بهخوبى وضع شده است و ثبات

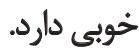

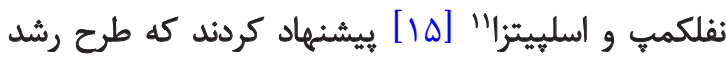

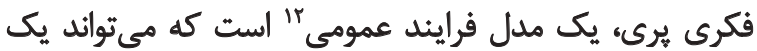

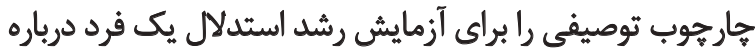

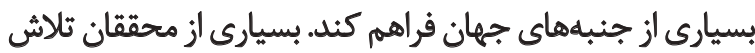

9. Epistemological

10. Shapiro

11. Knefelkamp \& Slepitza

12. General Process model 
كرامر و همكاران [1] براي اندازهكيرى همساني درونى يرسشنامه عقايد اصول اجتماعى با هر نوع عبارتئى (برايى مثال

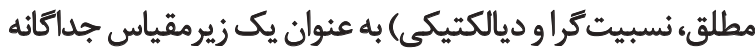

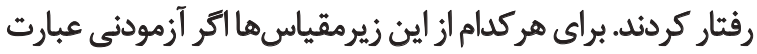

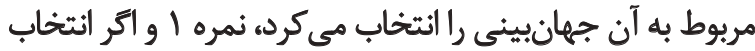

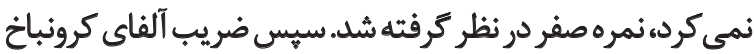

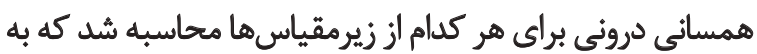

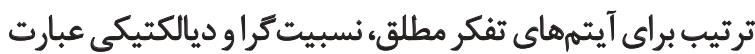

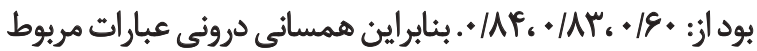

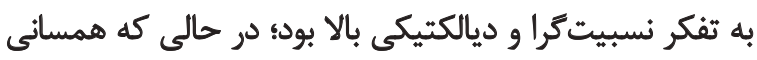

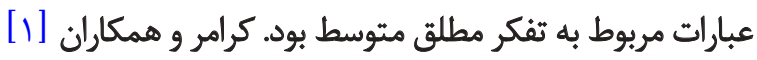

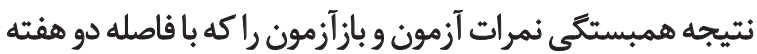

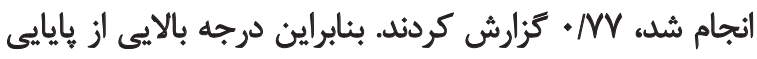

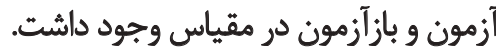

كرامر و همكاران [1] عنوان كرده بودند كه اكر قرار است

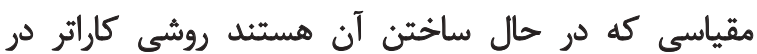

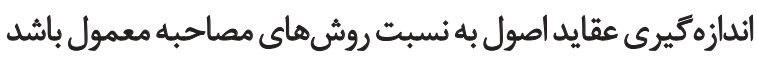

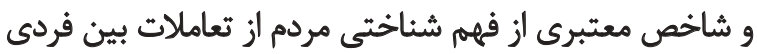

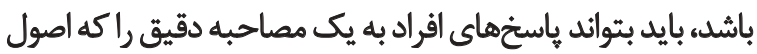

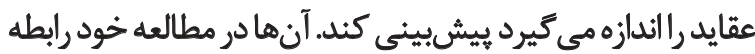

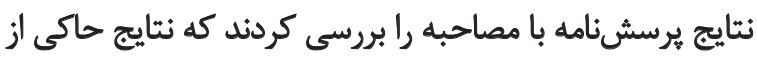

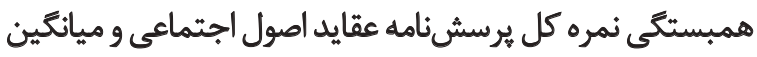

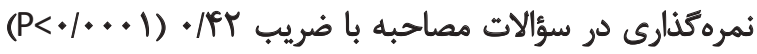

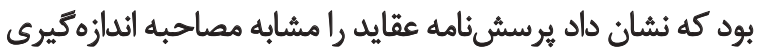

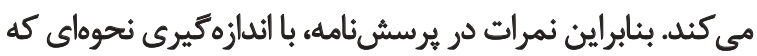

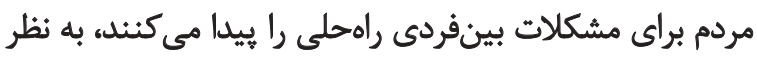

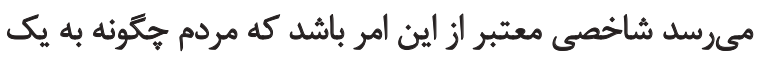
مصاحبه ساختاريافته ياسخ خواهئد دادي آمراد

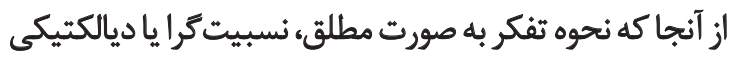

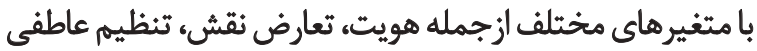

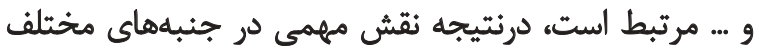

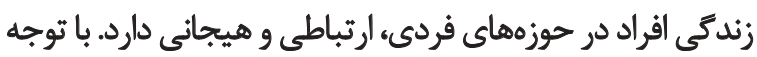

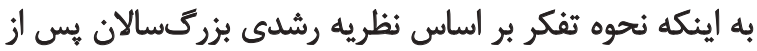

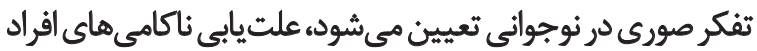

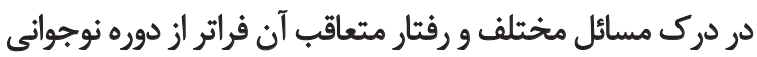

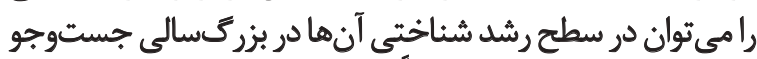

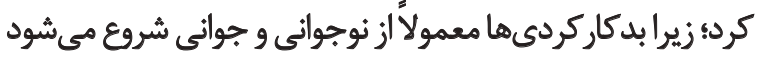

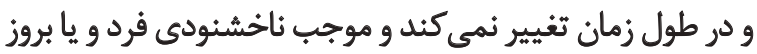

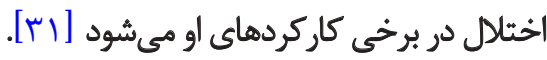

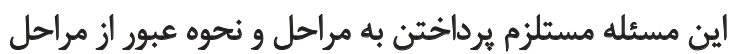

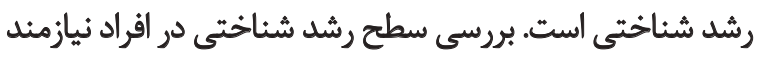

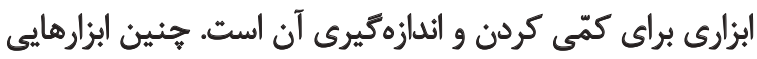

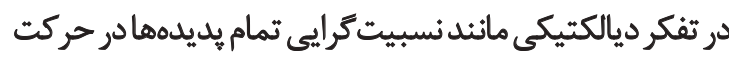

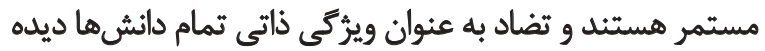

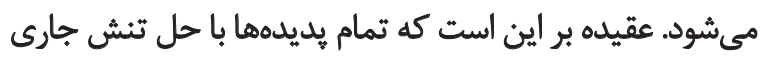

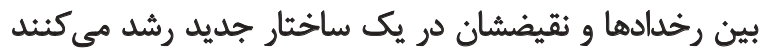

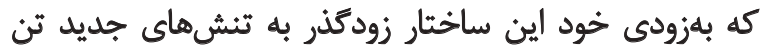

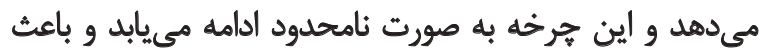

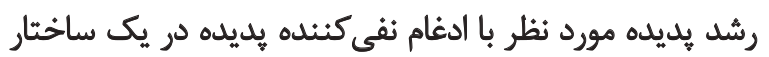

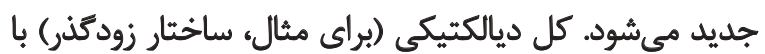

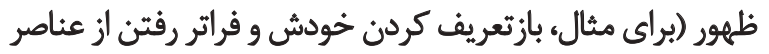

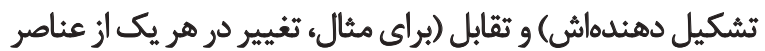

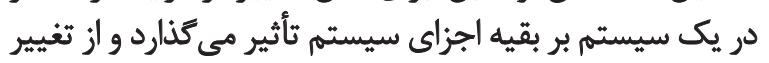

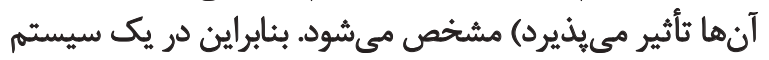

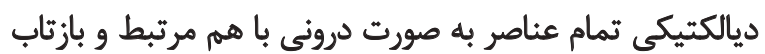

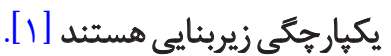

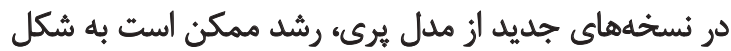

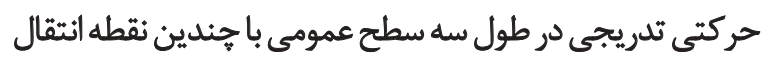

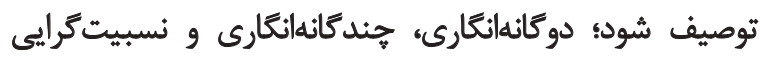

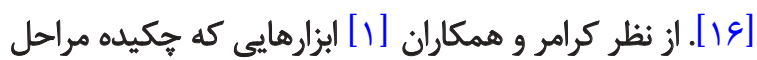

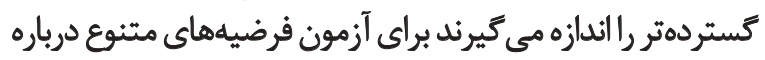

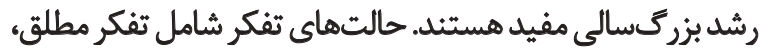

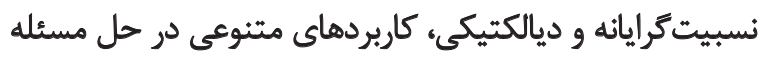

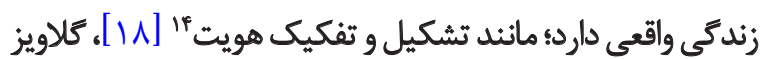

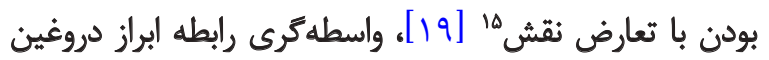

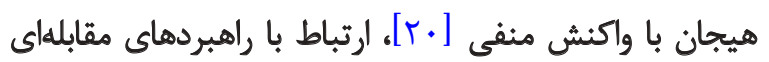

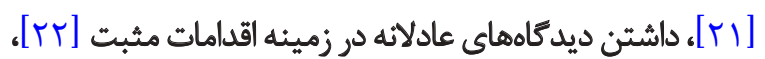

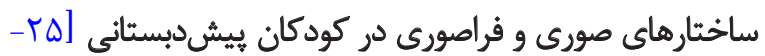

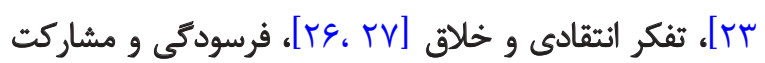

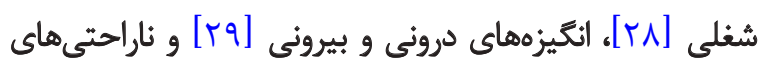

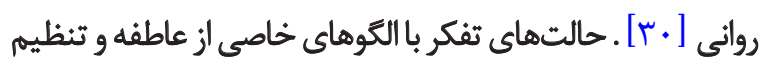

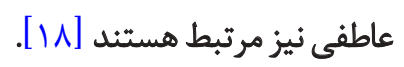

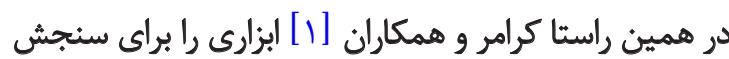

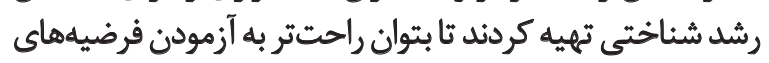

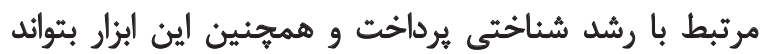

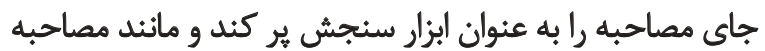

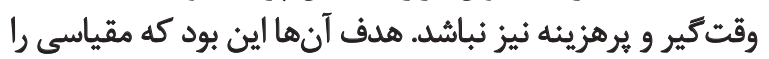

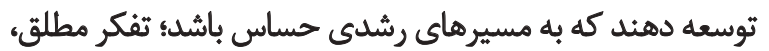

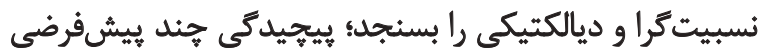

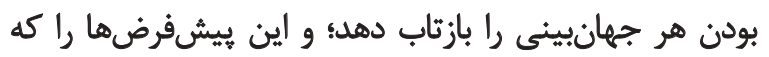

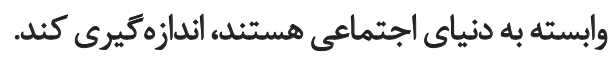

14. Identity formation and resolution

15. Grappling with role conflict 


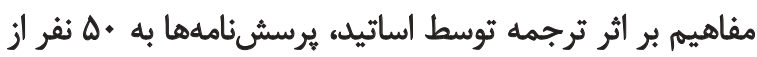

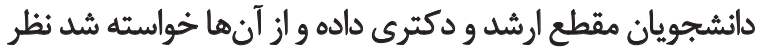

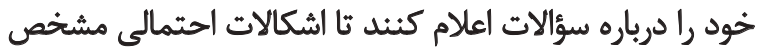

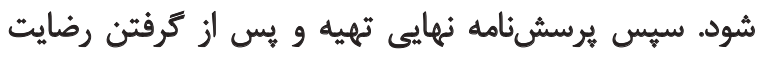

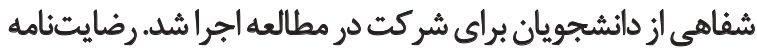

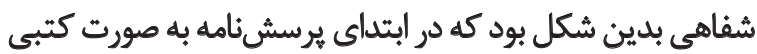

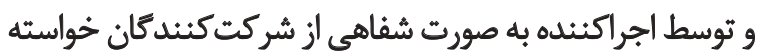

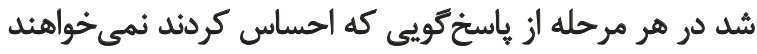

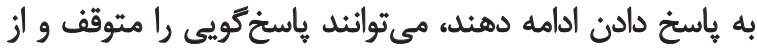
مصاحبه خارج شوند.

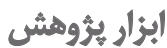

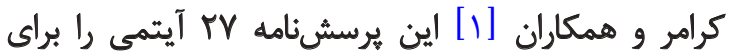

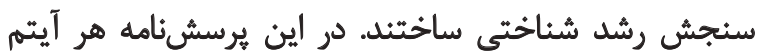

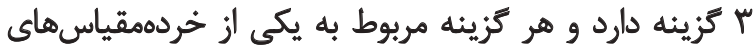

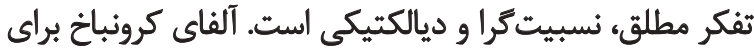

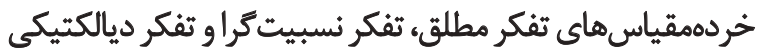

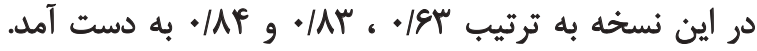

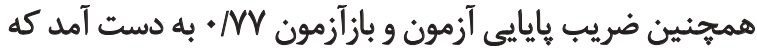

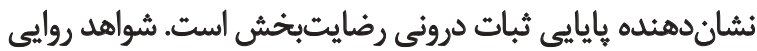

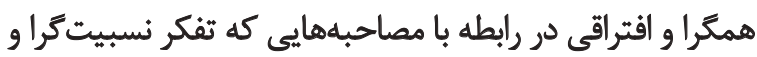

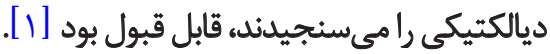

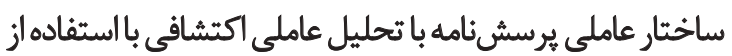

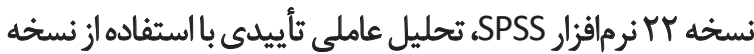

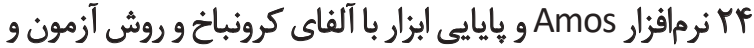

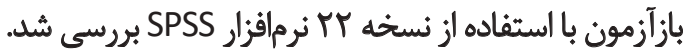

يافتهها

ويزّكىهاى جمعيت شناختى نمونه در جدول شماره آ آورده

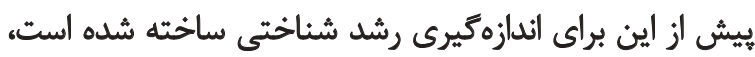

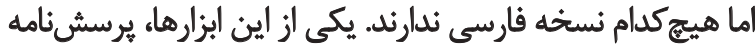

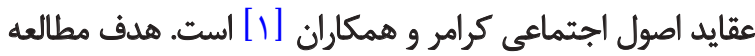
حاضر بررسى ساختار عاملى نسخه فارسى بر برسش اجتماعى و بررسى همسانى درونى مقياس در جامئى نامعه ايران است.

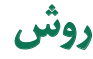

اين مطالعه توصيفي و از نوع ابزارسازى اعتبارسنجى است. جامعه أمارى، حجم نمونه و روش ثمونه يَيرى

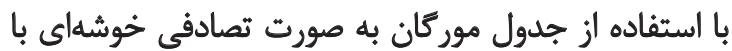

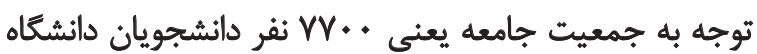

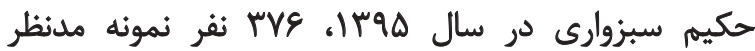

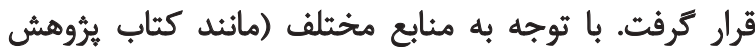

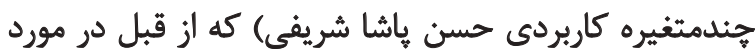

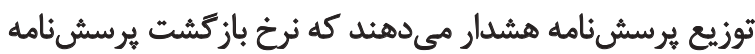

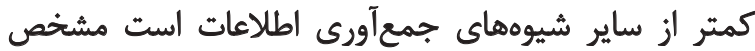

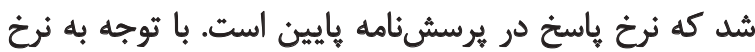

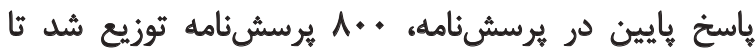

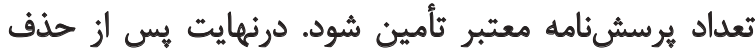

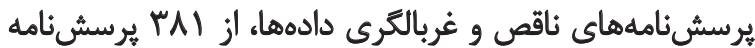
قابل قبول براى انجام تحيل استفاده شدي

$$
\text { روش اجراى يُؤهش }
$$

محققان اين يرسشنامه رابه زبان فارسى ترجمه كردند. سيس

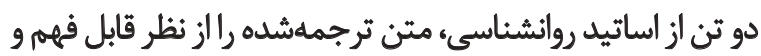

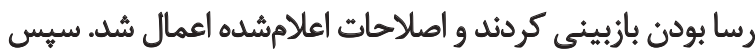

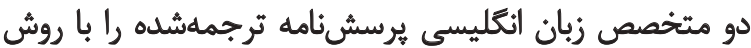

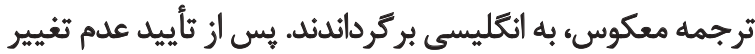

جدول ا. ويرّكى هاي جمعيتشناختى نمونه مطالعه

\begin{tabular}{|c|c|c|c|}
\hline درصد & قراوانى & \multicolumn{2}{|c|}{ متغير } \\
\hline pq/g & 119 & نختر & \\
\hline$\Delta+/ \kappa^{\epsilon}$ & 197 & يسر & جنس \\
\hline $\begin{array}{l}19 / \Delta \\
1.10\end{array}$ & $\begin{array}{l}m p \\
\text { p. }\end{array}$ & هثأهل & وضعيث تأهل \\
\hline$n e / s$ & rAF & كارشيناسي & \\
\hline repre & q & كاشناسي ارششد & تحصيلات \\
\hline 1 & $f$ & دكترا & \\
\hline
\end{tabular}




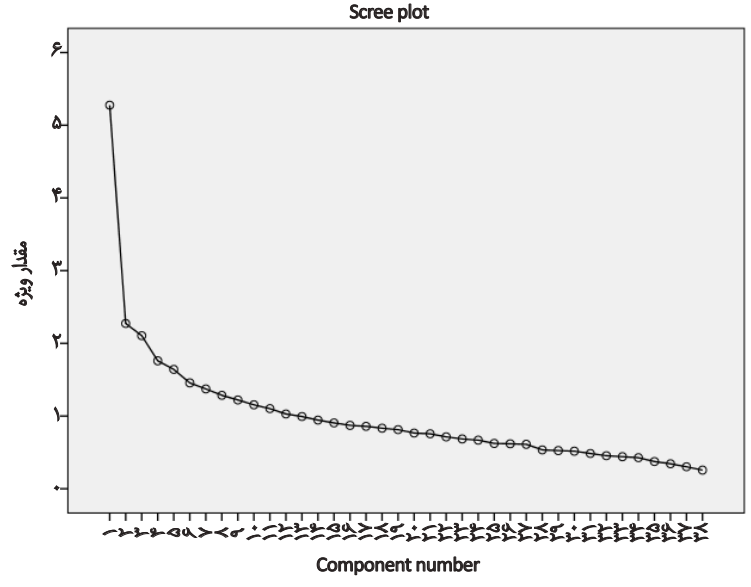

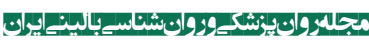

تصوير ا. نمودار اسكرى مربوط به ارزش ويزٔه تعيينشده توسط عوامل رشد

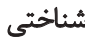

ارزش ويره عوامل در تصوير شماره ا نشان داده شده است. با

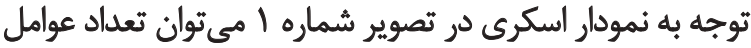

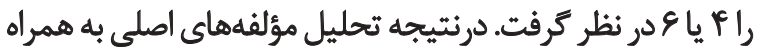

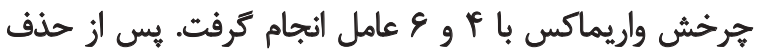

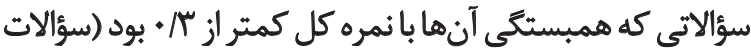

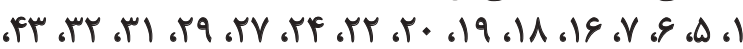

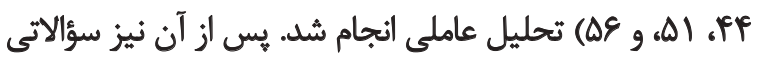

شده است. همانطور كه اطلاعات توصيفى جمعيتشناختى

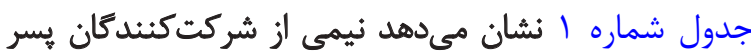

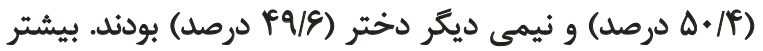

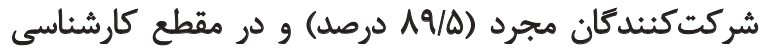

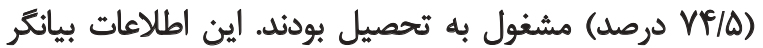

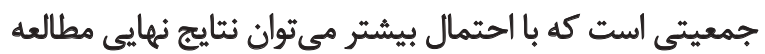
را به آنها تعميم داد.

براى بررسى اعتبار ساختار يرسشنامها إز تحليل عاملى اكتشافى إنى

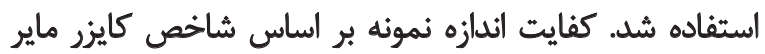

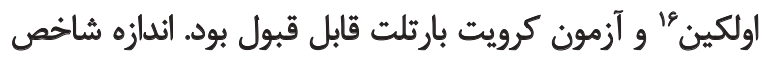

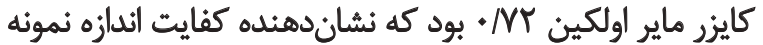

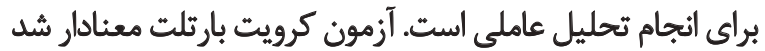

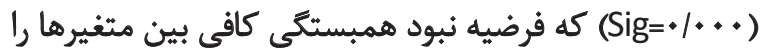

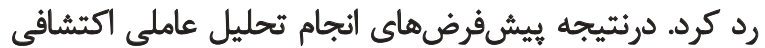

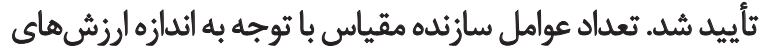

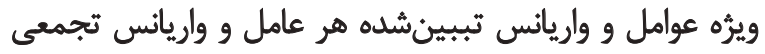

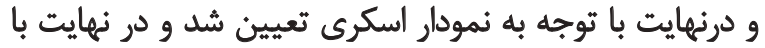

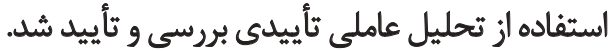
از تحليل مؤلفههاي اصلى با هرخش واريماكس براي تحليل

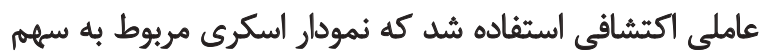
16. Kaiser-Meyer-Olkin (KMO)

جدول r. اندازههاى ارزش ويره، درصد واريانس هر عامل و درصد واريانس تجمعى راهحل جهارعاملى

\begin{tabular}{|c|c|c|c|}
\hline درصد واريانس تجمعى & درصد واريانس & ارزش ويزوه & عاملها \\
\hline$|F|+9$ & $|\psi|+8$ & $\Delta / T$ & 1 \\
\hline$r+/ M$ & s/Ir & r/Re & r \\
\hline$r \Delta / N$ & $\Delta / \& V$ & $r / 1$. & r \\
\hline$r . / \varepsilon$. & $P / M^{e}$ & I/ND & f \\
\hline
\end{tabular}

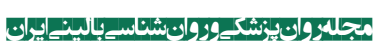

جدول r. اندازمهاى ارزٔش ويرّه، درصد واريائ هر عامل و درصد واريانس تجمعى راهحل ششعاملى

\begin{tabular}{|c|c|c|c|}
\hline درصد واريانس تجمعى & درصد واريانس & ارزش ويزه & عاملها \\
\hline $1 \% / 9 \varphi$ & Ir/ap & $\Delta / 1 \Delta$ & 1 \\
\hline$r .1 .1$ & $81 \cdot V$ & $t / \pi e^{2}$ & r \\
\hline$r \Delta / \& q$ & $\Delta / \& V$ & $r / l$. & r \\
\hline$r \cdot / \mu r$ & $P / M^{e}$ & INA & $r$ \\
\hline Tr/AV & $r / T r$ & V/94 & $ه$ \\
\hline rNVq & r/qY & $1 / 40$ & 8 \\
\hline
\end{tabular}


جدول P. آيتمهاى برسشئنه عقايد اصول اجتماعي و بارهاى عاملى آنها در مدل جهارعاملي

\begin{tabular}{|c|c|c|c|c|}
\hline عامل ع & عاهل "r & عامل r & عامل 1 & آيتمهها \\
\hline & & & . $/ P T$ & $A$ \\
\hline & & & $\cdot M T$ & if \\
\hline & & &. $\mid 11$ & 10 \\
\hline & & & . & rA \\
\hline & & & .10 & "ז \\
\hline & & & .10 & $m$ \\
\hline & & & .109 & ro \\
\hline & & & $\cdot / \pi T$ & rA \\
\hline & & & / / & rq \\
\hline & & &.$/ M$ & f. \\
\hline & & &.$/ \Delta P$ & il \\
\hline & & & $\cdot / r V$ & PQ \\
\hline & & &.$/ 4 V$ & is \\
\hline & & &.$|F|$ & $\Delta \Delta$ \\
\hline & & $+/ 40$ & & $r$ \\
\hline & & $\cdot / \mu$ & & 11 \\
\hline & & $+/ 8 \lambda$ & & $\pi$ \\
\hline & &.$/ 198$ & & II \\
\hline & &.$/ 18$ & & ro \\
\hline & & $\cdot(\Delta)^{P}$ & & re \\
\hline & & .119 & & $r$. \\
\hline & & .119 & & re \\
\hline & & $\cdot / \Delta A$ & & $\Delta P$ \\
\hline & $\cdot / \pi$ & & & $r$ \\
\hline & $\cdot|r|$ & & & 9 \\
\hline &.$/ F F$ & & & IV \\
\hline & $+/ \Delta+$ & & & PV \\
\hline & .10. & & & PA \\
\hline & . Mer & & & $19 q$ \\
\hline & . & & & $\Delta$. \\
\hline & .119 & & & Ar \\
\hline.$/ \pi T$ & & & & $r$ \\
\hline
\end{tabular}




\begin{tabular}{|c|c|c|c|c|}
\hline عامل ع & عامل r & عامل Y & عامل | & آيتهرها \\
\hline . & & & & 1. \\
\hline .18 & & & & $M$ \\
\hline$* / M V$ & & & & m \\
\hline 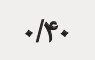 & & & & $r v$ \\
\hline .10 & & & & هr \\
\hline
\end{tabular}

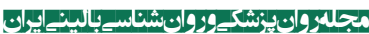

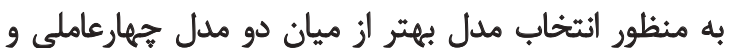

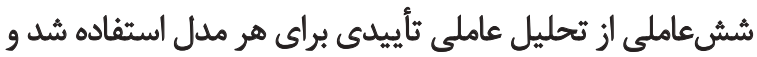

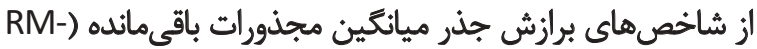

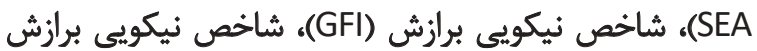

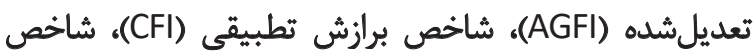

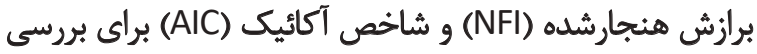

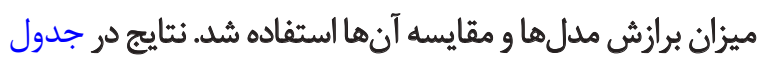

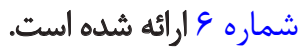

همه شاخصهاى برازش در جدول شماره ه حاكى از برازش
كه كمتر از س/ • روى عوامل بار داشتنده در هر كدام ازٔ راهحلهاي

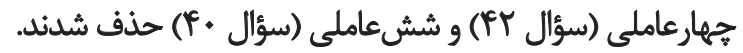
اندازههاى ارزش ويرُه، درصد واريانس هر عامل و واريانس

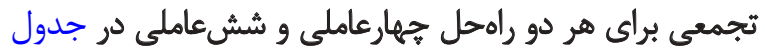

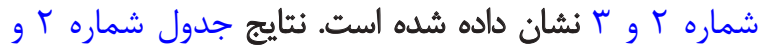

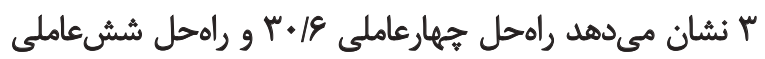

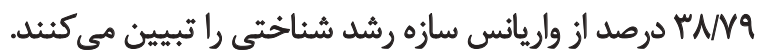

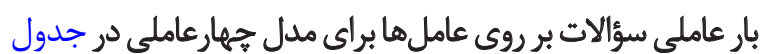
أ و براي مدل ششعاملى در جدول ها نشان داده شده است.

جدول هـ آيثمهاى يرسشنامه عقايد اصول اجتماعى و بارهاى عاملى آنها در مدل ششعاملى

\begin{tabular}{|c|c|c|c|c|c|c|}
\hline عامل 7 & عامل ه & عامل ع & عامل r & عامل r & عامل | & آيتم ها \\
\hline & & & & & . 19D & MA \\
\hline & & & & & 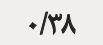 & זי \\
\hline & & & & & $.19 \pi^{\circ}$ & ra \\
\hline & & & & & . Arr & rA \\
\hline & & & & & .109 & rq \\
\hline & & & & & $.10 \Delta$ & pi \\
\hline & & & & & . & HT \\
\hline & & & & & . & iq \\
\hline & & & & ع & & f \\
\hline & & & & . & & 9 \\
\hline & & & &.$/ \mathrm{AS}$ & & 11 \\
\hline & & & & $\cdot|r|$ & & iv \\
\hline & & & &.$/ P r$ & & YD \\
\hline & & & & . $/ A T$ & & $M$ \\
\hline & & & & $\cdot / \mu$ & & Po \\
\hline & & & . & & & $r$ \\
\hline & & &.$|\Delta|$ & & & 1. \\
\hline & & & $\cdot / \Delta \Lambda$ & & & $\pi$ \\
\hline
\end{tabular}




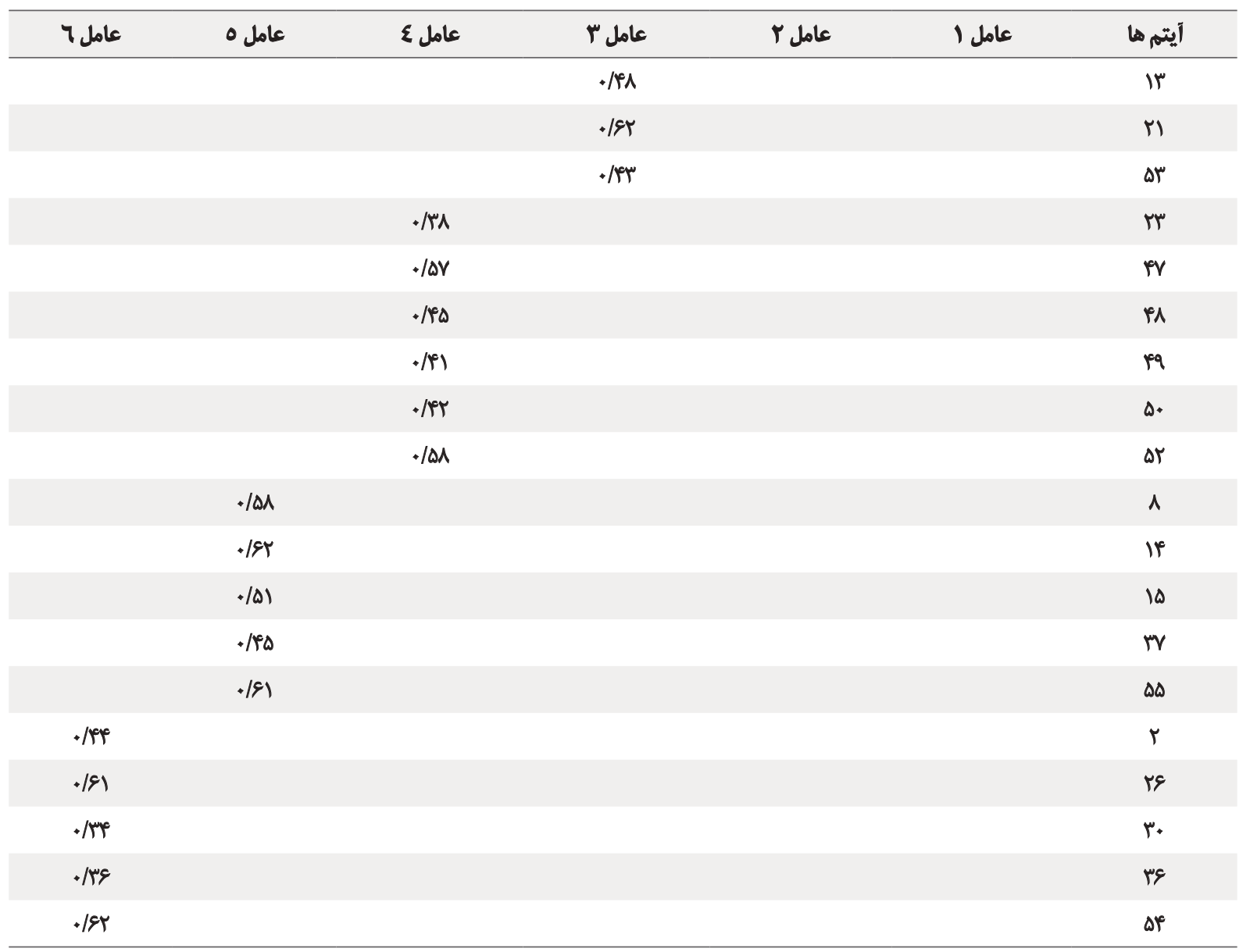

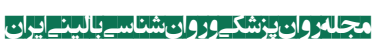

منحصر به فرد از مجموعه رفتارهاى تصادفى است. درنتيجه

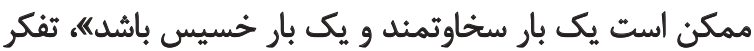

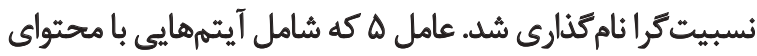

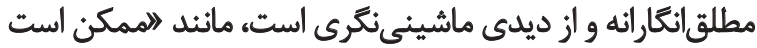

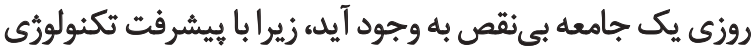

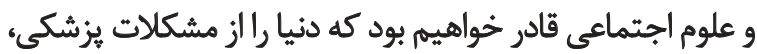

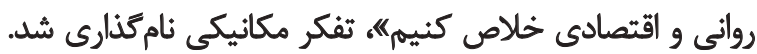

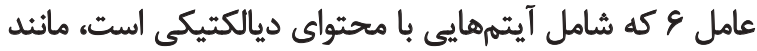

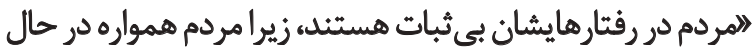

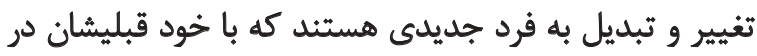

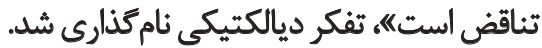
عامل ا شامل آيتمهايي است كه اعتقاد به ثبات افراد، نبود
بهتر مدل ششعاملى است. با توجه به برازش بهتر ساختار

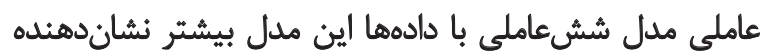

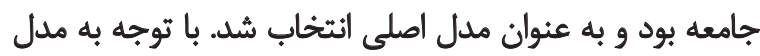

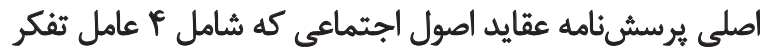

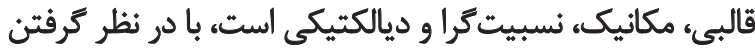

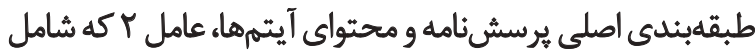

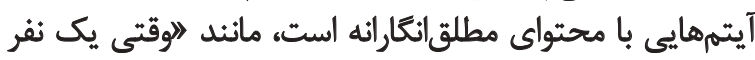

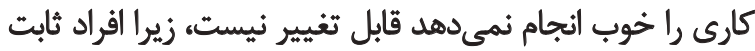

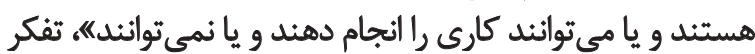
قالبى نام تمذارى شد. عامل f f كه شامل آيتمهايى با محتواي نسبيت كرايانه است، مانيد الرفتار افراد متغير است، زيرا هر شخص يت يك تركيب

جدول \&. شاخصهاى برازش مدل هاى جهارعاملى و ششعاملى

\begin{tabular}{|c|c|c|c|c|c|c|c|c|}
\hline AIC & AGFI & GFI & NFI & CFI & RMSEA & $x^{r}$ & Df & مدل \\
\hline IV8A & . 199 & $\cdot|A|$ &.$/ F \Delta$ & $\cdot / \Delta V$ & .1 .8 & $18+N M$ & grt & جهارعاملى \\
\hline Iane &.$/ 1$ & . AT &.$/ 0$ & . / & .1 .8 & IFANA. & 910 & شش عاملي \\
\hline
\end{tabular}


نثنايج كرامر و همكاران [ [ ] مبنى بر وجود درجه بالايى از بايايى آزمون و بازآزمون در مقياس هماهنگ است.

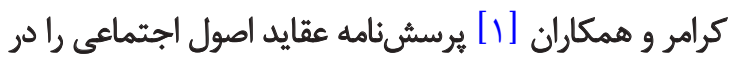

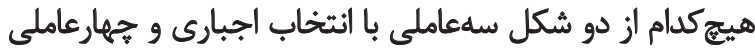

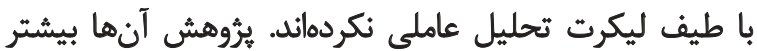

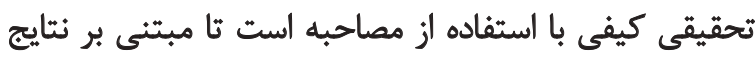

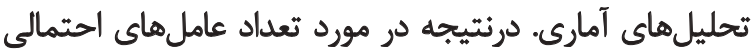

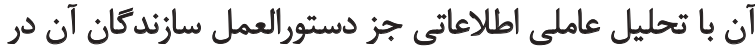

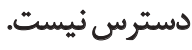

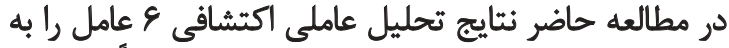

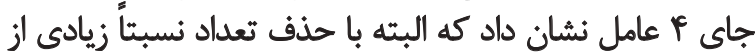

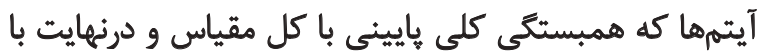

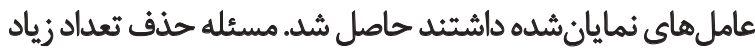

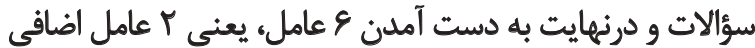
در مطالعه حاضر را مى ثوان به دو مسئله مرتبط دانست.

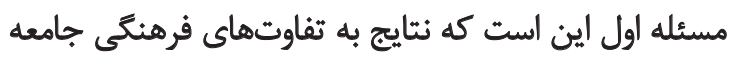

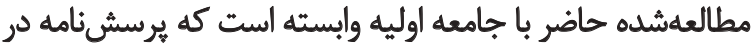

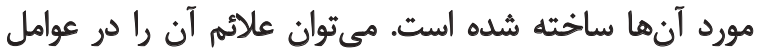

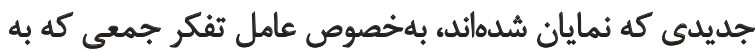

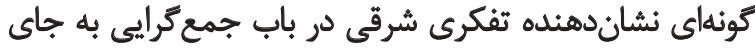

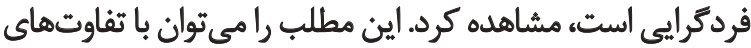

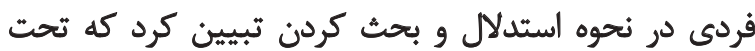

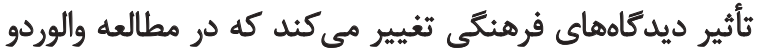

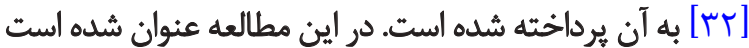

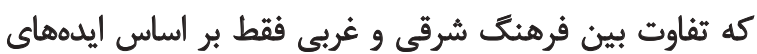

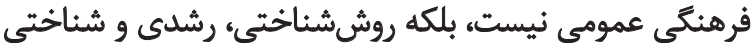

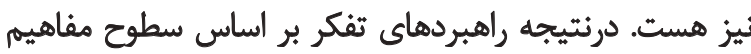

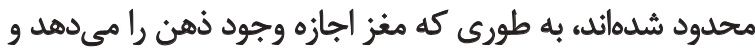

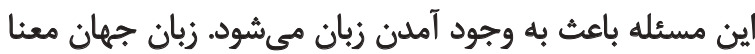

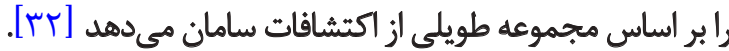
اين بررسىها درباره يرسشنامه اصلى انجام نشده است.

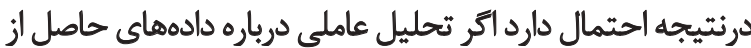

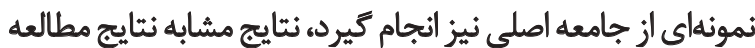

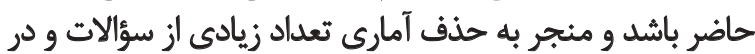

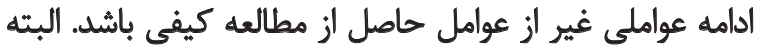

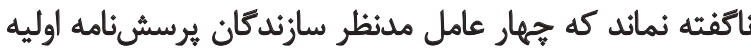

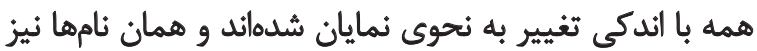

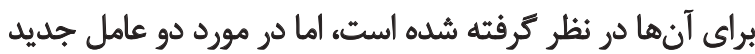

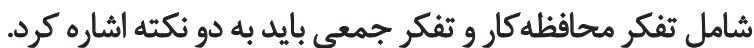
اول اينكه تفكر محافظه كار از لحاظ نوع به تروه تفكر مطلق تعلق دارد كه بيش از آن نيز شامل تفكر قالبى و مكانيكى بو بود

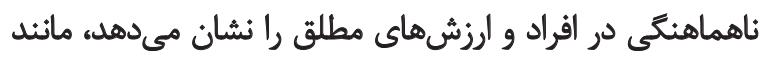

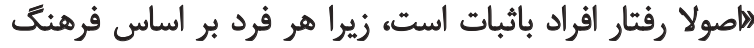

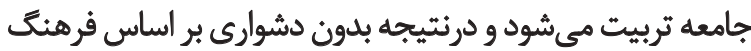

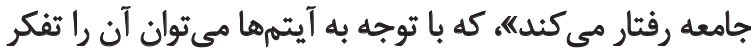

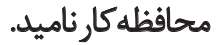

عامل r شامل آيتمهايى است كه نظر افراد را درباره نقش

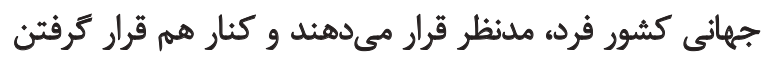

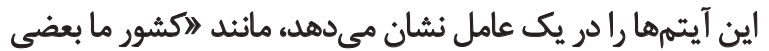

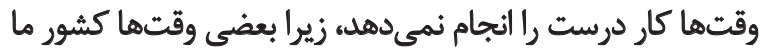

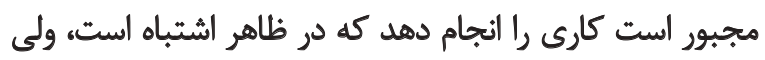

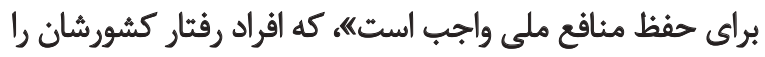

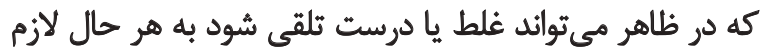

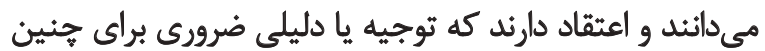

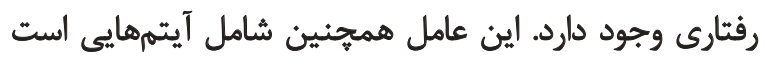

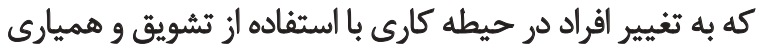

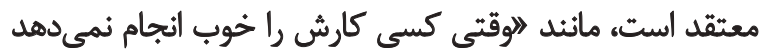

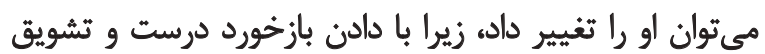

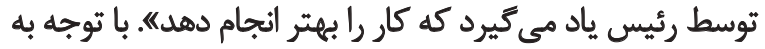

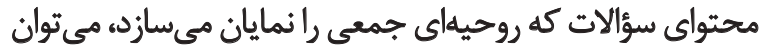

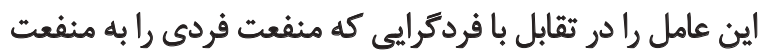
جمعى ترجيح مى دهد الففكر جمعى نائ ناميد.

همسانى درونى عواهل يرسشئامه با آلفاي كرونباخ اندازهكيرى

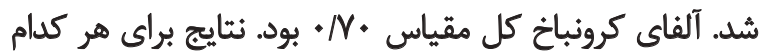

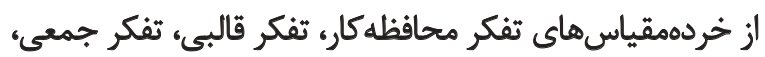

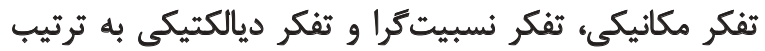
آزون

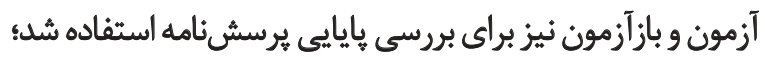

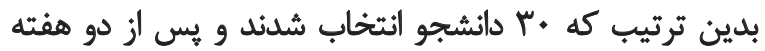

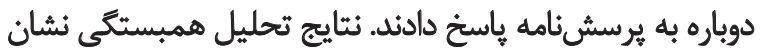

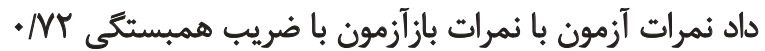

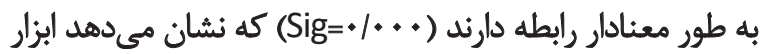

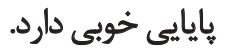

\section{ب ب0}

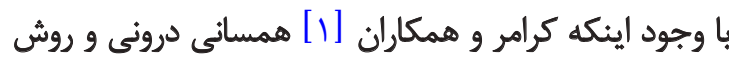

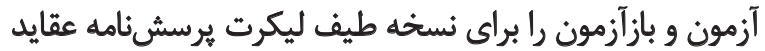

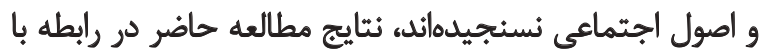

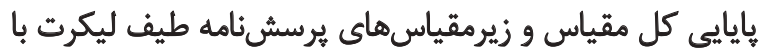

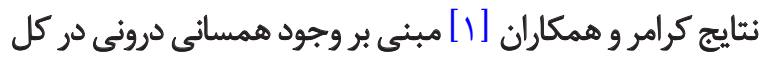

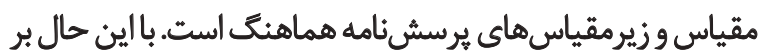

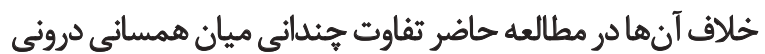

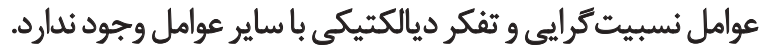

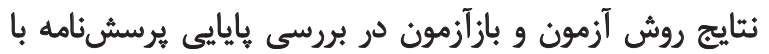


عوامل برسش تامه اصلى را نمايان مي كند. به طور كلى مي توان

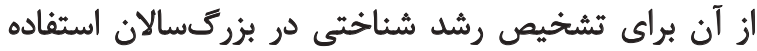
كرد و استفاده غربالكرى و بالينى دارد.

\section{نتيجليرى}

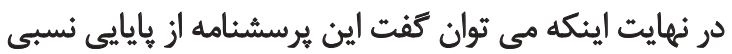

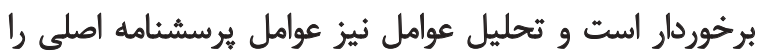

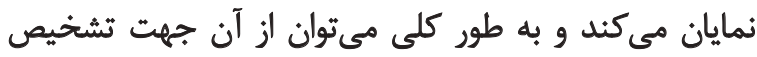

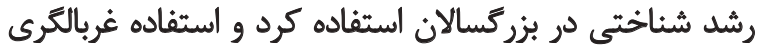

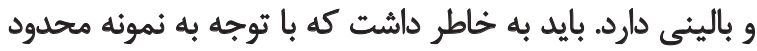

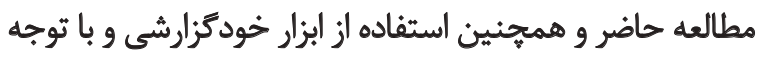
به ثفاوتهاى فرهنكى تعميم نتايج بايد با احتياط صورت بذئيرد

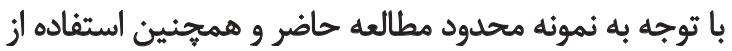

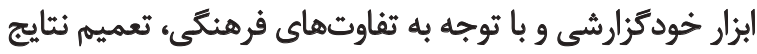

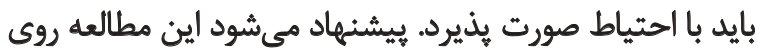

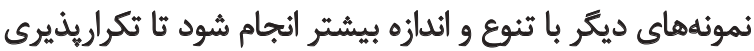

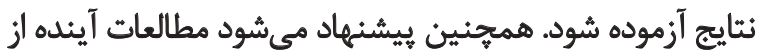

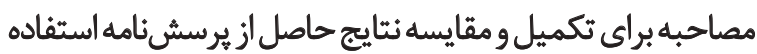

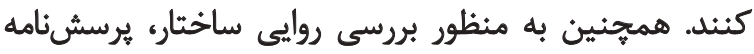
كنونى با يرسشنامههاى مشابه و متفاوت مقايسه شود.

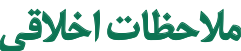

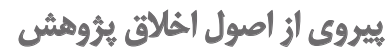

تمام شركت كنئدكان مطالعه با مفاد رضايت آكاهائه مطالعه

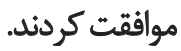

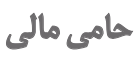

اين مطالعه هيج كونه حمايت مالى دريافت نكرده است.

$$
\text { تعارض مناقع }
$$

هيجّونه تعارض منافع ميان نويسندكان اين مطالعه وجود ندارد.

$$
\text { سباسكَّزازى }
$$

بدين وسيله از تمام شركت كنيدكان در مطالعه حاضر تشكر مىشود.
همجينين تفكر جمعى به نوعى بين تفكر مطلق و تفكر نسبيى تعلق

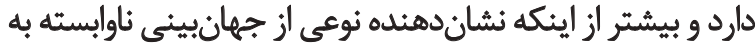

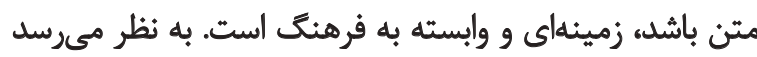

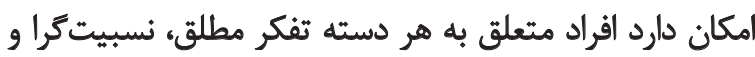

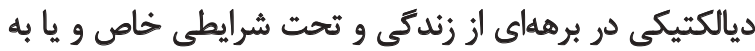

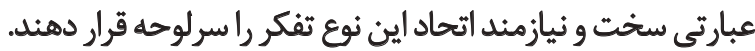

اين مطلب با يافتههاى مطالعه قاسيم و القهورى [سرس] مطابقت دارد كه در آن به انجام تحقيق كيفى در كشورهاى مانداف محافظه كار

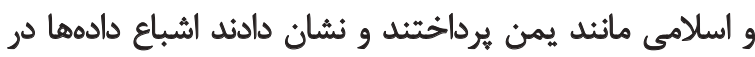

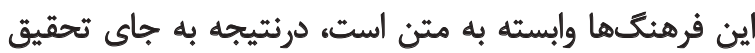

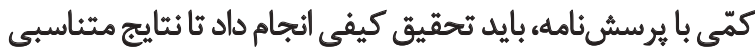

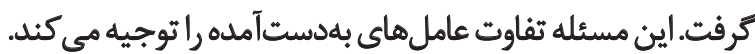

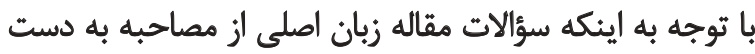

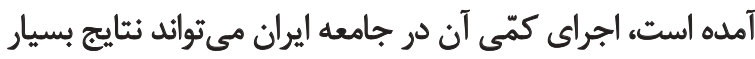

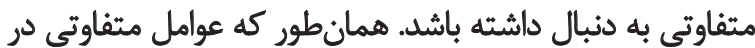

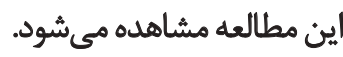

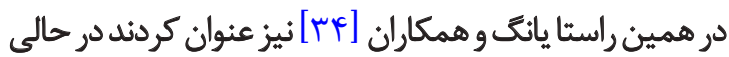

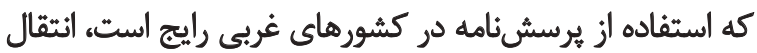

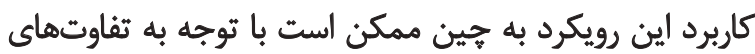

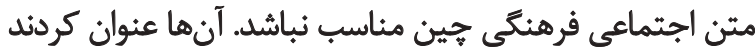

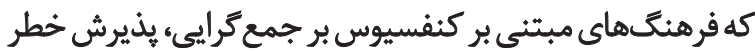

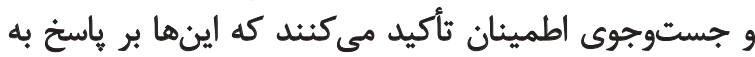

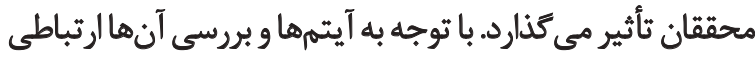

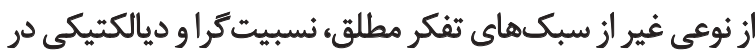

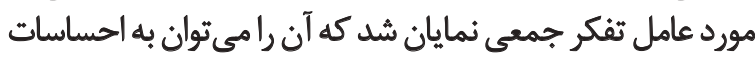

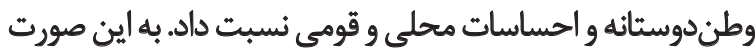

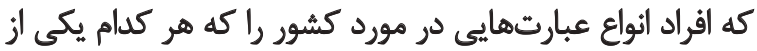

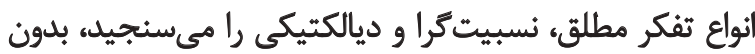

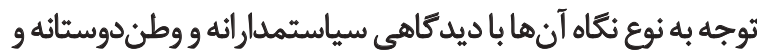

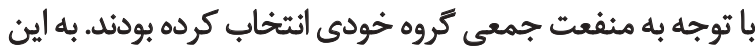

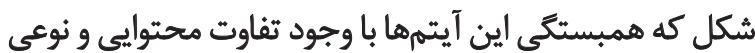

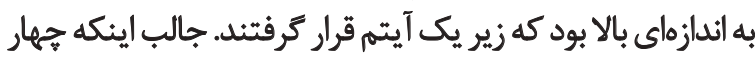

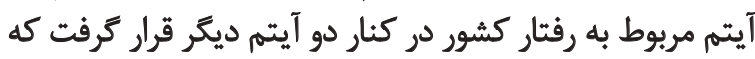

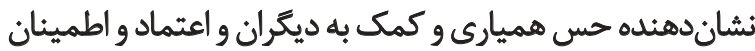

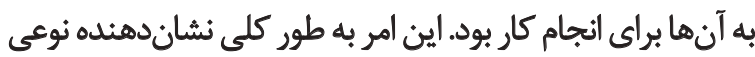

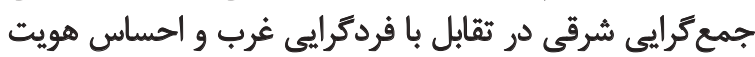
جمعى به جاى فردى و تفكرى عرفى است

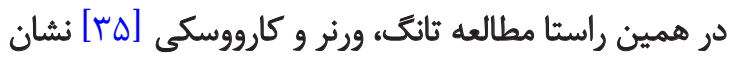

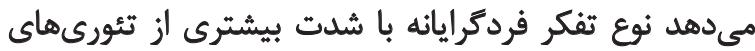

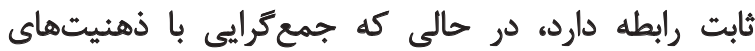

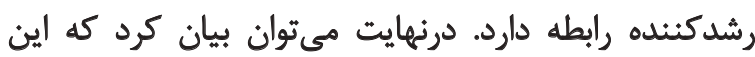
يرسشنامه از هايايى نسبى برخوردار است و تحليليل عوامل نيز بيز 


\section{References}

[1] Kramer DA, Kahlbaugh PE, Goldston RB. A measure of paradigm beliefs about the social world. Journal of Gerontology. 1992; 47(3):180-9. [DOI:10.1093/geronj/47.3.P180]

[2] Grove NP, Bretz SL. Perry's Scheme of Intellectual and Epistemological Development as a framework for describing student difficulties in learning organic chemistry. Chemistry Education Research and Practice. 2010; 11(3):207-11. [DOI:10.1039/C005469K]

[3] Frisancho S. The relationship between the primary appraisal of stress, dialectical thinking and moral dilemmas that threaten the self [Internet]. 2014 [Cited 2014 May 16]. Available from: http:/ / blog.pucp.edu.pe/blog/wp-content/uploads/ sites/32/2006/11/Stress-and-Thinking.pdf

[4] Csikszentmihalyi M, Rathunde K. The development of the person: An experiential perspective on the ontogenesis of psychological complexity. In: Csikszentmihalyi M, editor. Applications of Flow in Human Development and Education. Berlin: Springer; 2014.

[5] Fischer KW. Dynamic cycles of cognitive and brain development: Measuring growth in mind, brain, and education. In: Battro AM, Fischer KW, Léna PJ, editors. The Educated Brain: Essays in Neuroeducation. Cambridge: Cambridge University Press; 2008. p. 127-50. [DOI:10.1017/CBO9780511489907.010]

[6] Harvey OJ. Experience structure \& adaptability. Berlin: Springer; 2013.

[7] Dwyer CP, Hogan MJ, Stewart I. An integrated critical thinking framework for the $21^{\text {st }}$ century. Thinking Skills and Creativity. 2014; 12:43-52. [DOI:10.1016/j.tsc.2013.12.004]

[8] Lane JD, Wellman HM, Olson SL, LaBounty J, Kerr DC. Theory of mind and emotion understanding predict moral development in early childhood. British Journal of Developmental Psychology. 2010; 28(4):871-89. [DOI:10.1348/026151009X483056] [PMID]

[9] Syed M, Seiffge-Krenke I. Personality development from adolescence to emerging adulthood: Linking trajectories of ego development to the family context and identity formation. Journal of Personality and Social Psychology. 2013; 104(2):371. [DOI:10.1037/ a0030070] [PMID]

[10] Riegel KF. Foundations of dialectical psychology. Amsterdam: Academic Press; 1979.

[11] Grossmann I, Na J, Varnum ME, Kitayama S, Nisbett RE. A route to well-being: Intelligence versus wise reasoning. Journal of Experimental Psychology: General. 2013; 142(3):944. [DOI:10.1037/a0029560] [PMID] [PMCID]

[12] Black S, Allen JD. Part 3: College Student Development. The Reference Librarian. 2017; 58(3):1-5. [DOI:10.1080/02763877.2 016.1276505]

[13] Neely ME. Epistemological and writing beliefs in a first-year college writing course: Exploring shifts across a semester and relationships with argument quality. Journal of Writing Research. 2014; 6(2). [DOI:10.17239/jowr-2014.06.02.3]

[14] Wang Q, Capous D, Koh JBK, Hou Y. Past and future episodic thinking in middle childhood. Journal of Cognition and Development. 2014; 15(4):625-43. [DOI:10.1080/15248372.2013.784977]
[15] Brock R. The challenge of plural conceptions. Studies in Science Education. 2016; 52(1):81-92. [DOI:10.1080/03057267.2014.964515]

[16] Kramer DA, Bopp MJ. Transformation in clinical and developmental psychology. New York: Springer-Verlag; 2013. [DOI:10.1007/978-1-4612-3594-1]

[17] Basseches M. The development of dialectical thinking as an approach to integration. Integral Review: A Transdisciplinary and Transcultural Journal For New Thought, Research, and Praxis. 2005; 1(1):47.

[18] Sadr MM. The role of personality traits and perceived parenting styles in predicting cognitive developmen. International Journal of Applied Behavioral Sciences. 2016; 3(2):27-34.

[19] Chang YYC, Chiou W-B. Diversity beliefs and postformal thinking in late adolescence: A cognitive basis of multicultural literacy. Asia Pacific Education Review. 2014; 15(4):585-92. [DOI:10.1007/s12564-014-9345-6]

[20] Hideg I, Kleef GA. When expressions of fake emotions elicit negative reactions: The role of observers' dialectical thinking. Journal of Organizational Behavior. 2017; 38(8):1196-212. [DOI:10.1002/job.2196]

[21] Cheng C. Dialectical thinking and coping flexibility: A multimethod approach. British Journal of Psychology. 2009; 77(2):47194. [DOI:10.1111/j.1467-6494.2008.00555.x]

[22] Hideg I, Ferris DL. Dialectical thinking and fairness-based perspectives of affirmative action. Journal of Applied Psychology. 2017; 102(5):782. [DOI: 10.1037/apl0000207] [PMID]

[23] Shiyan IB. Dialectical thinking as a mechanism of construction of possible relations for senior pre-school children. ProcediaSocial and Behavioral Sciences. 2013; 86:308-11. [DOI:10.1016/j. sbspro.2013.08.569]

[24] Shiyan OA, Kokontseva EV. Interconnection of the ability to cooperate with peers and development of dialectical thinking of senior pre-school children. Procedia-Social and Behavioral Sciences. 2014; 146:83-8. [DOI:10.1016/j.sbspro.2014.08.090]

[25] Veraksa N. Dialectic and formal structures in cognition of preschool children. Procedia-Social and Behavioral Sciences. 2014; 146:118-23. [DOI:10.1016/j.sbspro.2014.08.097]

[26] Tanaka J, Gilliland B. Critical thinking instruction in Eenglish for academic purposes writing courses: A Dialectical Thinking Approach. TESOL Journal. 2017; 8(3):657-74. [DOI:10.1002/tesj.291]

[27] Arlin Pk. Dialectical thinking: further implications for creative thinking. In: Runco M editor. Encyclopedia of Creativity (Second edition). Amsterdam: Academic Press; 2011. [DOI:10.1016/B9780-12-375038-9.00072-8]

[28] Leon MR, Halbesleben JR, Paustian-Underdahl SC. A dialectical perspective on burnout and engagement. Burnout Research 2015; 2(2-3):87-96. [DOI:10.1016/j.burn.2015.06.002]

[29] Li Y, Sheldon KM, Liu R. Dialectical thinking moderates the effect of extrinsic motivation on intrinsic motivation. Learning and Individual Differences. 2015; 39:89-95. [DOI:10.1016/j.lindif.2015.03.019]

[30] Pang J, Keh HT, Li X, Maheswaran D. Every coin has two sides: The effects of dialectical thinking and attitudinal ambivalence on psychological discomfort and consumer choice. Journal of Consumer Psychology. 2017; 27(2):218-30. [DOI:10.1016/j.jcps.2016.10.001] 
[31] Soraya S, Kamalzadeh L, Nayeri V, Bayat E, Alavi K, Shariat SV. Factor structure of personality inventory for DSM-5 (PID-5) in an Iranian sample. Iranian Journal of Psychiatry and Clinical Psychology. 2017; 22(4):308-17. [DOI:10.18869/nirp.ijpcp.22.4.308]

[32] Vallverdú J. Brains, language and the argumentative mind in Western and Eastern societies. The fertile differences between Western-Eastern argumentative traditions. Progress in Biophysics and Molecular Biology. 2017; 131:424-31. [DOI:10.1016/j.pbioł molbio.2017.09.002]

[33] Kasim A, Al-Gahuri HA. Overcoming challenges in qualitative inquiry within a conservative society. Tourism Management. 2015; 50:124-9. [DOI:10.1016/j.tourman.2015.01.004]

[34] Yang J, Ryan C, Zhang L. The use of questionnaires in Chinese tourism research. Annals of Tourism Research. 2012; 39(3):1690-3. [DOI:10.1016/j.annals.2012.02.002]

[35] Tang M, Werner C, Karwowski M. Differences in creative mindset between Germany and Poland: The mediating effect of individualism and collectivism. Thinking Skills and Creativity. 2016; 21:31-40. [DOI:10.1016/j.tsc.2016.05.004] 
